\title{
Fast Tracking the Fundamental component in Synchrophasors applications using the Recursive Corrected Phase Wavelet Transform
}

\author{
C. D. P. Crovato ${ }^{1}$, V. Souza ${ }^{1}$, G. S. Wojichowski ${ }^{1}$ and A. A. Susin ${ }^{2}$ \\ ${ }^{1}$ Embrasul Industria Eletrônica \\ Av. Bahia 684, CEP: 90240551, São Geraldo, Porto Alegre - RS (Brasil) \\ Phone number:+55 + (51) 3358-4000, e-mail: \{cesar, vinicius, guilherme\}@embrasul.com.br \\ ${ }^{2}$ Departamento de Engenharia Elétrica (DELET) \\ Universidade Federal do Rio Grande do Sul (UFRGS) \\ Av. Osvaldo Aranha, 103 CEP: 90035-190, Bom Fim, Porto Alegre, RS (Brasil) \\ Phone/Fax number:+55 + (51) 3308-3515 / 3308-3293, e-mail: altamiro@ufrgs.br
}

\begin{abstract}
This paper presents the use of Recursive Corrected Phase Wavelet Transform to track harmonic components (always present in Power Systems), in order to determine the fundamental frequency "as soon as possible", to provide appropriate input values to a non-linear algorithm that can accurately approach in only one cycle the amplitude and phase of the fundamental frequency. This technique is useful in real-time synchrophasors applications, specially for load identification and characterization. Based on the deepest descend method, the algorithm has less computational effort in comparison with phased locked loop.
\end{abstract}

\section{Key words}

Deepest descent algorithm, Phase Corrected Wavelet Transform, phasor estimation, Power Quality, recursive algorithm.

\section{Introduction}

As the electric power grid continues to expand and as transmission lines are pushed to their operating limits, the dynamic operation of the power system has become more of a concern and has become more difficult to model accurately. In addition, in order to prevent wide scale cascading outages, the ability to control real-time systems is turning into a need [1].

It is very desirable to be able to "measure" the system state, increase the refresh rate of the phasor estimators (amplitude, phase and frequency). The limit of doing this (without overlapping), is to make one estimation per fundamental cycle [2].

On the other hand, it is known that there are many different methods for tracking the spectral components, several papers discuss the use of Sliding Discrete Fourier Transform (SDFT) [3],[4]. Also, the SDFT has been used as a tool for visualization of time-varying harmonics and inter-harmonics, providing a better way to understand time dependent Power Quality (PQ) parameters [5]-[8].
Recent studies suggest that this kind of approach can help the detection and classification of events and be useful for load identification and characterization [9]-[10].

The SDFT formally implements the Short-Time Discrete Fourier Transform (STDFT) and some classic papers present a complete comparison between the well-known windows in terms of their frequency domain properties [11]-[13]. In previous work [14], this comparison was reviewed, in order to choose a window that can assist to overcome the inherent limitation of rectangular window, for asynchronous sampling rate and/or presence of interharmonics. Also propose the use of a polynomial (and frequency-dependent shape) sliding window, that formally implement the Recursive Corrected Phase Wavelet Transform (RCPWT), which can track the fundamental component in four cycles [10].

The input-output relationship of many devices should be described by a polynomial or Taylor series. This type of device are common in the electrical system and the nonlinear distortion generated by them, is always present, originating $h$ order harmonic components of fundamental [15]. During a fundamental cycle, many cycles of harmonics has passed. With this characteristic, first estimating the harmonics frequencies is more effective than estimating the fundamental frequency, since the estimations converge more quickly when frequency dependent kernels are used.

In this work we use the RCPWT as a tool to mainly determine: the frequency of a high order harmonic, the frequency of fundamental component and finally, using an adapted deepest descent method, find the phase and amplitude of the fundamental component.

\section{The Recursive Phase Corrected Wavelet Transform}

Unlike the SDFT, when using Gaussian Window (GW) as a sliding window, its additional parameter $\sigma$ (standard deviation parameter) allows shape modification 
according to the inverse of the frequency [16]. Choosing the standard deviation parameter properly, the convergence time for trace spectral components can be reduced as the tracking frequency increases; it makes frequency-dependent windows, like GW, more attractive. Moreover, to achieve efficiency, the GW should allow a recursive implementation, a classic way to obtain this, is through the IIR-filter representation; but as a closed form for GW in the z-domain doesn't exists, some authors propose the use of polynomial approach [17]-[19]. The GW can be approximate by many different techniques [20], but in general they need non-causal filters which the implementation can compromise the real time requirements. Moreover, it has been recently published a work that gets similar results to a $\mathrm{GW}$, through the implementation of the RPCWT [10], using a polynomial window. The main advantages of polynomial windows are the low computational complexity and ability to easily change their frequency response modifying the values of their coefficients in the time domain [21].

The "instantaneous spectra" (1) can be estimate through a complex demodulation followed by a convolution (the equation are in continuous domain, for simplicity, because it is more compact in this way). The analytical deduction of the Polynomial Window and its discrete recursive implementation was introduced in [10].

$$
X\left(\tau, f_{n}\right)=\left(e^{-j 2 \cdot \pi f} n^{\tau} x(\tau)\right) g\left(\tau, f_{n}\right)
$$

Where $x(\tau)$ is the voltage signal at $\tau$ time, $f_{n}$ is the tracking frequency, $j=\sqrt{-1}$ and $g\left(t, f_{n}\right)$ is the window function defined as:

$$
g\left(t, f_{n}\right)=\frac{o f_{n} e^{-o f_{n} t}}{2160}\left(4\left(o f_{n} t\right)^{6}-12\left(o f_{n} t\right)^{5}+30\left(o f_{n} t\right)^{4}\right)
$$

To be an "admissible" RPCWT Kernel, $o=2 \pi / \sqrt{3}[16]$.

From the obtained complex numbers in (1), it is possible to determine the estimation for the instantaneous amplitude and phase. Using the definition in [22] the instantaneous frequency can be found from the derivate of the instantaneous phase:

$$
\operatorname{IF}\left(\tau, f_{n}\right)=\frac{1}{2 \pi} \frac{\partial}{\partial \tau}\left(2 \pi f_{n} \tau+\operatorname{angle}\left(X\left(\tau, f_{n}\right)\right)\right)
$$

\section{The adapted deepest descent method applied}

Be $x[m]$ a sampled voltage signal of the power grid:

$$
x[m]=\operatorname{Acos}\left(2 \pi f T_{s} m+\varphi\right)+v[m]
$$

Where $v[m]$ includes the effect of all the harmonics, inter-harmonics, transients and noise. $A, f, \varphi$ are amplitude, frequency and phase of the fundamental component, respectively; $m$ is the sample index, $T_{s}$ is the sampling time.

Let $\tilde{A}, \tilde{f}, \tilde{\varphi}$ the amplitude, frequency and phase estimations of the fundamental component and $\boldsymbol{e}(\tilde{A}, \tilde{f}, \tilde{\varphi})$ the residual error in one cycle of the fundamental component defined as follows:

Where:

$$
\boldsymbol{e}(\tilde{A}, \tilde{f}, \tilde{\varphi})=\boldsymbol{x}-\tilde{A} \cos \left(2 \pi \tilde{f} T_{s} \boldsymbol{m}+\tilde{\varphi}\right)
$$

$$
\begin{aligned}
& \boldsymbol{x}=[x[m] \ldots x[m+N-1]]^{T}, \\
& \boldsymbol{e}=[e[m] \ldots e[m+N-1]]^{T}, \\
& \boldsymbol{m}=[m \ldots m+N-1]^{T}
\end{aligned}
$$

The estimations are optimal $\{\tilde{A}, \tilde{f}, \tilde{\varphi}\}_{\mathrm{o}}$ when the residual error has its minimum mean square error (MSE) value:

$$
\{\tilde{A}, \tilde{f}, \tilde{\varphi}\}_{\mathrm{o}} \approx\{A, f, \varphi\} \mid \min _{\text {glob }}\{\operatorname{MSE}\{\boldsymbol{e}(\tilde{A}, \tilde{f}, \tilde{\varphi})\}\}
$$

The function $\operatorname{MSE}\{\boldsymbol{e}(A, f, \varphi)\}$ can have many local minima which turns the search for the optimal solution a heavy computational task.

The Fig. 1, Fig. 2 and Fig. 3 show the surface of the MSE with at least one parameter remaining constant and varying the other two: $\left.\operatorname{MSE}\{\mathrm{e}(\mathrm{A}, \mathrm{f}, \varphi)\}\right|_{\mathrm{f}=\text { const }}$ $\left.\operatorname{MSE}\{\mathrm{e}(\mathrm{A}, \mathrm{f}, \varphi)\}\right|_{\mathrm{A}=\text { const }}$ and $\left.\operatorname{MSE}\{\mathrm{e}(\mathrm{A}, \mathrm{f}, \varphi)\}\right|_{\varphi=\text { const }}$ respectively, for a distorted synthetic signal $x[\mathrm{~m}]$.

$$
x[m]=1 \cdot \cos \left(2 \pi 60 T_{s} m+40^{\circ}\right)+v[m]
$$

where $v[\mathrm{~m}]$ contains $3^{\text {th }}$ to $13^{\text {th }}$ harmonics with different (and random) amplitudes and phases. $T_{s}=1 / 7680 \mathrm{~s}$.

The frequency remaining constant (as in Fig .1) is the only case that the MSE does not have local minima.

Considering that the frequency of the fundamental component is known, the search for the optimal estimations for $A$ and $\varphi$ becomes a simple task, because $\left.\operatorname{MSE}\{\boldsymbol{e}(A, f, \varphi)\}\right|_{f \approx \tilde{f}}$ has a unique global minimum. To find the best estimations in a few iterations, with the steepest descent method, it is very desirable to start with initial values near to the optimal ones.

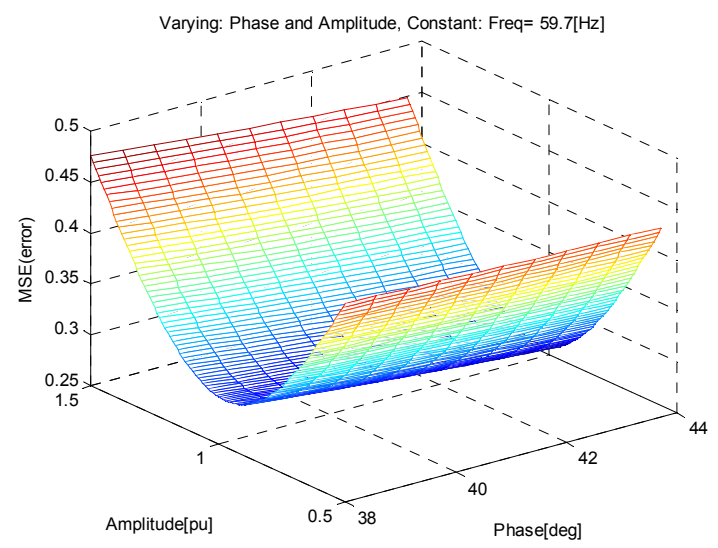

Fig. 1. Mean Square Error, for fixed Freq. $\left.\operatorname{MSE}\{\mathrm{e}\}\right|_{\mathrm{f}=\text { const }}$ 


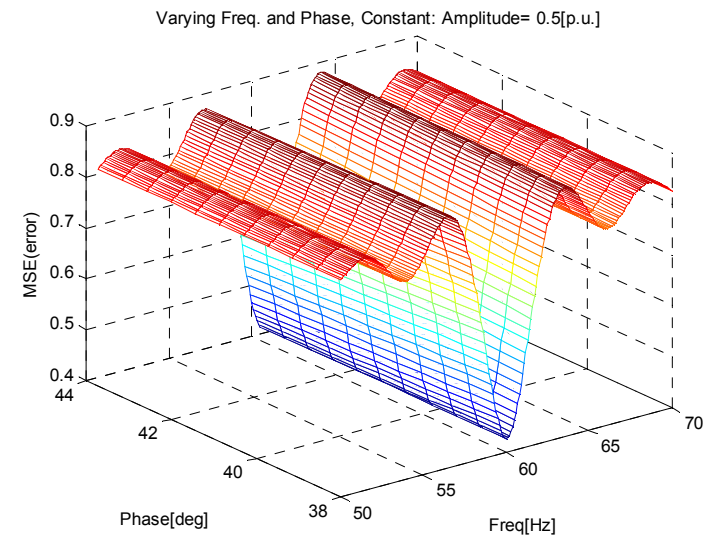

Fig. 2. Mean Square Error, for fixed Freq. $\left.M S E\{e\}\right|_{A=c o n s t}$

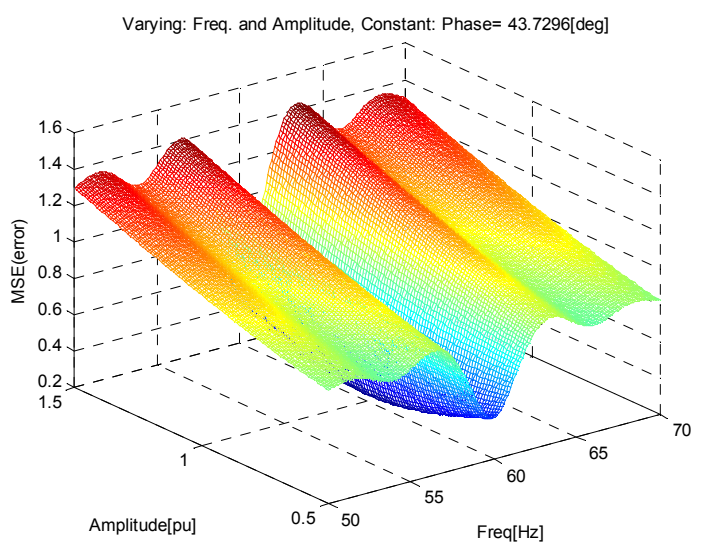

Fig. 3. Mean Square Error, for fixed Freq. $\left.M S E\{e\}\right|_{\varphi=c o n s t}$

\section{Description of the overall system}

Fig. 4. General view of the three main algorithms, exemplifying the proposed scheme

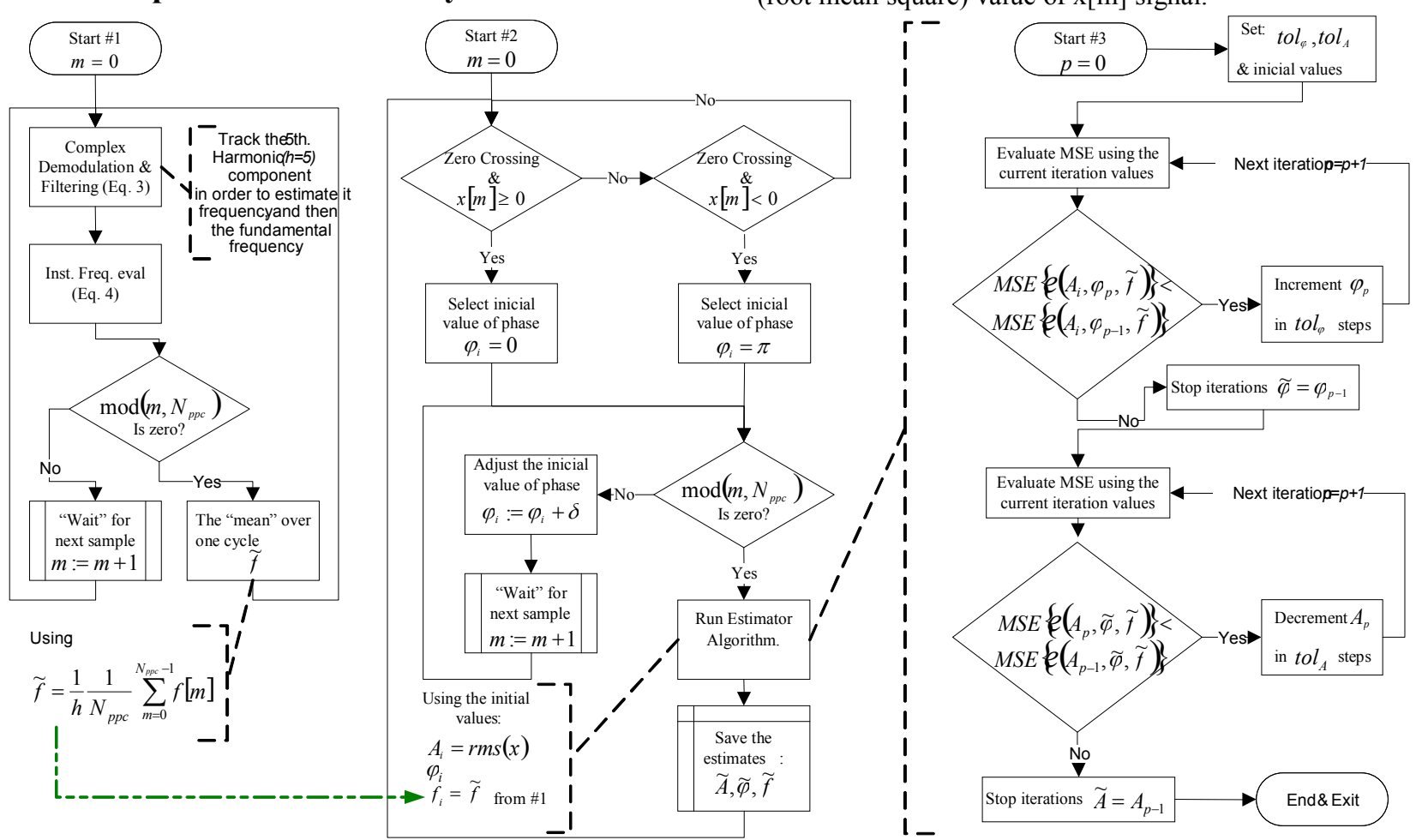

Figure 3 resumes the three mains algorithms (\#1, \#2, \#3 from the left to the right).

When the algorithm \#1 starts, the first step is to track the frequency of the $5^{\text {th }}$ harmonic $(h=5)$ using the discrete version of RCPWT (1). This operation can be done directly by complex demodulation following by linear convolution (1) and thereafter, the instantaneous frequency is estimated using (3). In [10] these procedures are described in discrete domain.

This global procedure is repeated until the number of samples reached the number of points per cycle $\left(N_{p p c}\right)$ of the nominal fundamental frequency. Then, instantaneous frequency of the $5^{\text {th }}$ harmonic are averaged over the cycle period and divided by 5 , in order to get the fundamental estimation $(\tilde{f})$.

The algorithm \#2 (Fig. 3) describes the procedure to estimate amplitude and phase of the fundamental component. The first step of the algorithm is to determine the zero crossing point of the $x[\mathrm{~m}]$ signal. If the zero crossing occurs from negative to positive we define a first approximation phase as zero $\left(\varphi_{i}=0\right)$ otherwise we define as $\pi\left(\varphi_{i}=2 \pi\right)$.

After that, the algorithm waits for one complete cycle of the signal to run the estimator (algorithm \#3). Meanwhile, the phase approximation is incremented by $\delta$ for each point until the signal reaches a complete cycle. The $\delta$ is calculated according to $\left(N_{p p c}=\right.$ points per cycle $)$ :

$$
\delta=2 \pi / N_{p p c}
$$

The estimator algorithm (algorithm \#3) receives as parameters the frequency approximation $f_{i}=\widetilde{f}_{i}$, as calculated in algorithm $\# 1$, the phase approximation $\varphi_{i}$ and the amplitude approximation Ai defined as the rms (root mean square) value of $\mathrm{x}[\mathrm{m}]$ signal. 
Then it returns the estimated values for amplitude $(\tilde{A})$ and phase $(\tilde{\varphi})$ of the fundamental.

The algorithm \#3 describes the amplitude and phase estimator. At the beginning of the algorithm, five parameters must be set: amplitude, phase and frequency values calculated in algorithms \#1 and \#2 and tolerance values for amplitude $\left(t o l_{\varphi}\right)$ and phase $\left(t o l_{A}\right)$. The last two parameters are needed to reduce the search range. The estimation of phase is run first. The values of amplitude $\left(A_{i}\right)$ and frequency $(\tilde{f})$ are kept constant and the phase $\left(\varphi_{p}\right)$ varies. The phase starts at $\varphi_{p}=\varphi_{i}-t_{o l}$ and ends at $\varphi_{p}=\varphi_{i}+\operatorname{tol}_{\varphi}$, where $p$ represents the iteration index.

The MSE (mean square error) is calculated for each $\varphi_{p}$ until a minimum value is found. The first approximation of the phase parameter is a good approximation so the search is done only near the optimal point, reducing the time spent on search. The algorithm stops search when the difference between the MSE value for the present and the last phase is positive, indicating the optimal value was found. The phase estimation is set as $\tilde{\varphi}=\varphi_{p-1}$.

The estimation of amplitude runs the same way the estimation of phase. The initial values of phase $(\tilde{\varphi})$ and frequency $(\tilde{f})$ are set constant and the amplitude $\left(A_{p}\right)$ varies. The phase varies from $A_{p}=A_{i}+t o l_{A}$ to $A_{p}=$ $A_{i}-t o l_{A}$, where $p$ represents the index of amplitude. The algorithm stops search when the difference between the MSE value for the present and the last amplitude is positive, indicating the optimal value was found. The amplitude estimation is set as $\tilde{A}=A_{p-1}$.

\section{Experiments and Results}

Many synthetic signals were used for performance evaluation, all of them with $f=60 \mathrm{~Hz}$ and low SNR as $15 \mathrm{~dB}$, under different distorted condition. To graphically illustrate the Fig. 5 represents many of them.

Also, some real data from IEEE database [23] was used too. To graphically illustrate, the Fig. 6 represents many of them. The sampling frequency was $\mathrm{Fs}_{S}=7680 \mathrm{~Hz}, 128$ point per cycle, $T_{s}=1 / \mathrm{Fs}$.

The synthetic signal, in Fig. 5, is defined as:

$$
\begin{gathered}
x[m]=1 \cos \left(2 \pi 60 T_{s} m+40^{\circ}\right)+ \\
0.2 \cos \left(2 \pi 300 T_{s} m+8^{\circ}\right)
\end{gathered}
$$

With an overall fall (sag) from $\mathrm{x}[\mathrm{m}]$ to $\mathrm{x}[\mathrm{m}] / 2$ at $m=600$ and overall up (swell) two cycles after.

The real signal in Fig. 6, is full of time-varying harmonics. The Phasor Estimation (algorithm \#2,\#3) for every cycle of the fundamental component are shown in Table I. It is clear that for the $x$ signal the error remaining is less than $0.2 \%$. The performance was significantly reduced during transients, and the estimation shows out of range deviations and must be flagged. This procedure is out of the scope of this work and will be omitted.

During tests the authors uses an EPLL (enhanced phase locked loop) based on filter bank proposed in [24] for computational effort comparative purposes. The EPLL must be fine tuned previously with tree convergence parameters according to the sampling rate. On the proposed technique the only design parameter is the desired tolerance, and it is independent of the sampling rate.

Also, in the EPLL the number of math operations and table searching are greater than the proposed non-linear technique for the same number of points per fundamental cycle, as showed in Table II for tracking (9).
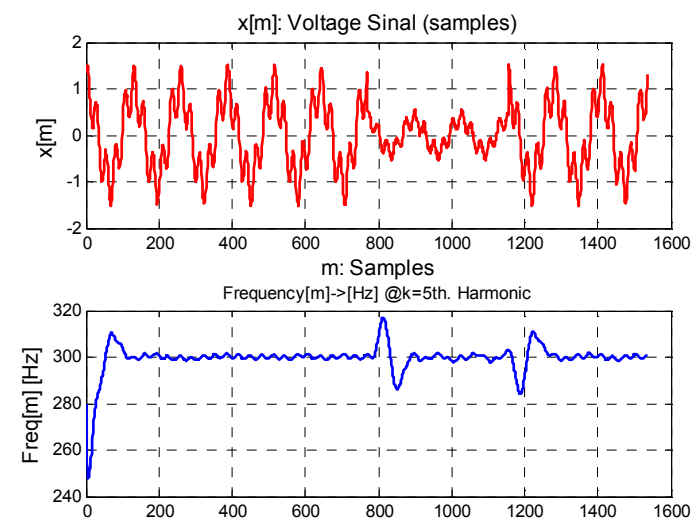

Fig. 5. Synthetic voltage signal $x[\mathrm{~m}]$ and the frequency estimation of its $5^{\text {th }}$ harmonic (algorithm \#1).
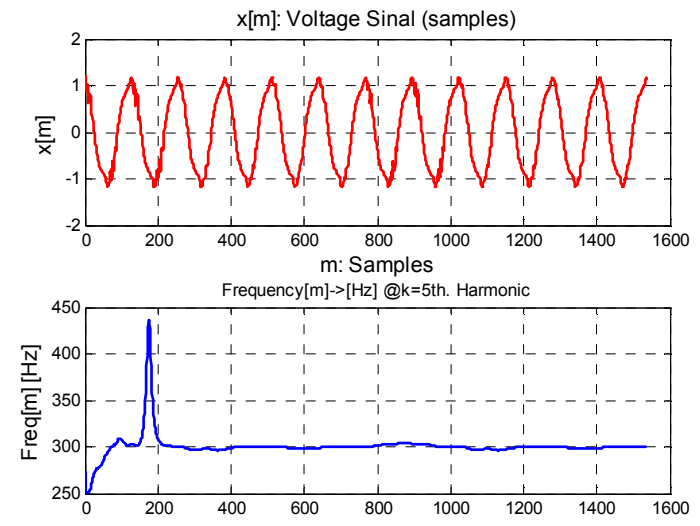

Fig. 6. Real voltage signal $y[\mathrm{~m}]$ and the frequency estimation of its $5^{\text {th }}$ harmonic (algorithm \#1).

Table I. - Phasor Estimation for a synthetic signal $\boldsymbol{x}$, and for a real signal $\boldsymbol{y}$.

\begin{tabular}{|l|l|l|l|l|l|l|}
\hline CYCLE & \multicolumn{2}{|l|}{ FREQUENCY } & \multicolumn{2}{c|}{ AMPLIT. } & \multicolumn{2}{c|}{ PHASE } \\
\cline { 2 - 7 } NUM & $\boldsymbol{x}$ & $\boldsymbol{y}$ & $\boldsymbol{x}$ & $\boldsymbol{y}$ & $\boldsymbol{x}$ & $\boldsymbol{Y}$ \\
\hline 1 & 58.48 & 60.20 & .97 & 1.116 & 40.1 & 18.39 \\
\hline 2 & 60.01 & 62.10 & 1.0 & 0.896 & 39.8 & 18.18 \\
\hline 3 & 60.02 & 59.22 & .982 & 1.106 & 39.9 & 17.75 \\
\hline 4 & 59.99 & 59.99 & .991 & 1.116 & 40.3 & 17.48 \\
\hline 5 & 59.96 & 59.67 & .988 & 1.116 & 39.7 & 17.04 \\
\hline 6 & 59.99 & 59.83 & .987 & 1.116 & 39.7 & 16.77 \\
\hline 7 & 60.01 & 61.18 & .487 & 1.056 & 37.5 & 18.33 \\
\hline 8 & 59.81 & 60.09 & .498 & 1.076 & 39.3 & 18.18 \\
\hline 9 & 60.17 & 59.25 & .508 & 1.026 & 39.5 & 17.75 \\
\hline 10 & 59.99 & 59.99 & 1.02 & 1.106 & 40.8 & 17.48 \\
\hline 11 & 60.00 & 59.67 & 1.01 & 1.096 & 40.2 & 17.04 \\
\hline 12 & 60.03 & 59.80 & .987 & 1.106 & 40.4 & 16.78 \\
\hline
\end{tabular}


Table II. - Comparative computational effort for the proposed technique and EPLL based [24], for $\mathrm{Fs}=7680 \mathrm{~Hz}$.

\begin{tabular}{|l|c|c|}
\hline \multirow{2}{*}{ OPPERATION } & \multicolumn{2}{|c|}{ METHOD } \\
\cline { 2 - 3 } & EPLL & Algorithms \#2,\#3 \\
\hline Addition & 640 & 384 \\
\hline Multiplication & 896 & 512 \\
\hline cos, sin function & 256 & 128 \\
\hline
\end{tabular}

The computational effort of algorithm \#1 has not been showed on Table II, because it is highly dependent of the implementation method of the IIR filtering.

\section{Conclusion}

This work has shown a technique based on the Recursive Corrected Phase Wavelet Transform to track harmonic components, in order to determine the fundamental frequency, to provide appropriate input values to a nonlinear algorithm that can approach the fundamental phasor. This research showed that the choice of the initial values of the estimations reduce drastically the number of iterations to reach convergence, and has proposed methods to determine near-optimal guessed values. The algorithm presents some limitation during transients, in this cases flagging is recommended, future work will deal with this issue. The method presents good results and can be useful in synchrophasors applications, and/or load characterization.

\section{Acknowledgement}

The authors thank FINEP (protocol 4941) for financial support.

\section{References}

[1] Sykes, J.; Koellner, K.; Premerlani, W.; Kasztenny, B.; Adamiak, M., "Synchrophasors: A primer and practical applications," Power Systems Conference: Advanced Metering, Protection, Control, Communication, and Distributed Resources, 2007. PSC 2007 , vol., no., pp.213-240, 13-16 March 2007.

[2] Bollen, Math H. J. / Gu, Irene. Signal Processing of Power Quality Disturbances- IEEE Press Series on Power Engineering - August 2006 ISBN-13: 978-0-471-73168-9 - John Wiley Sons.

[3] Eric Jacobsen, Richard Lyons, An update to the sliding DFT, IEEE Signal Processing Mag., Jan. 2004.

[4] Jaroslaw Gronczynski, Recursive Fourier transform algorithms with integrated windowing, Signal Processing 87 (2007) 1003-1013.

[5] Nakano K, Ota Y, Ukai H, Nakamura K, Fujita H. Frequency Detection Method Based on Recursive DFT Algorithm", Proceedings of the 14th Power Systems Computation. Conference (PSCC '02), Paper 5, pp. 1-7, Sevilla, Espanha, 2002

[6] Paulo Silveira, Carlos Duque, Thomas Baldwin, Paulo Ribeiro. Time-Varying Power Harmonic Decomposition using Sliding-Window DFT. ICHQP2008. The 13th IEEE International Conference on Harmonics and Quality of Power. Wollongong, New South Wales, Australia. 28th Sep. 1st Oct., 2008

[7] Carvalho, J.R. Duque, C.A. Baldwin, T.L. Ribeiro, P.F. A DFT-based approach for efficient harmonic/interharmonic analysis under time-varying conditions. Power and Energy Society General Meeting - Conversion and Delivery of Electrical Energy in the 21st Century, 2008 IEEE 20-24 July 2008

[8] Xin Limin Xu Weisheng Yu Youling A Fast Harmonic Detection Method Based on Recursive DFT Electronic Measurement and Instruments, 2007. ICEMI apos;07. 8th International Conference on. Volume , Issue , Aug. 16 2007-July 182007 Page(s):3-972 - 3-976

[9] Carvalho, J. R.; Gomes, P. H.; Duque, C. A.; Ribeiro, M. V.; Cerqueira, A. S.; Szczupak, J. PLL Based Multirate Harmonic Estimation Power Engineering Society General Meeting, 2007. IEEE Volume, Issue , 24-28 June 2007 Page(s): $1-6$.

[10] C.D.P.C Crovato, A. Susin. A Kernel for Recursive Corrected-Phase Wavelet Transform for Power Quality Assestment. ICHQP2008. The 13th IEEE International Conference on Harmonics and Quality of Power. Wollongong, New South Wales, Australia. 28th Sep. 1st Oct., 2008.

[11] F.J. Harris, On the use of windows for harmonic analysis with discrete transform, Proc. IEEE 66 (1) (January 1978) 51-83.

[12] N.C. Geçkinli, D. Yavuz, Some novel windows and a concise tutorial comparison of window families, IEEE Trans. Acoust. Speech Signal Process. 26 (6) (December 1978) 501-507.

[13] A.H. Nuttal, Some windows with very good sidelobe behaviour, IEEE Trans. Acoust. Speech Signal Process. 29 (1) (February 1981) 84-91

[14] C.D.P.C Crovato, A.Schuck Jr., A. Susin. "Frequency Dependent Windowing For Tracking Harmonics And Interharmonics In Power Systems: Survey And The Recursive Corrected Phase Wavelet Transform Approach". COBEP - Brazilian Power Electronics Conference 2009- Bonito-Brazil.

[15] K.C. Agrawal, Industrial Power Engineering Handbook, Newnes 1 Edition. London (2001), pp.999.

[16] Oppenheim, A. V., R. W. Schafer, and J. R. Buck. Discrete-Time Signal Processing . 2nd ed. Upper Saddle River, NJ: Prentice Hall, 1999

[17] Deriche R. 1992. Recursively implementing the Gaussian and its derivatives. In: Proceedings of the Second International Conference on Image Processing, Singapore, September 1992. p. 263-7.

[18] Jin, JS, Gao Y. Recursive implementation of LoG Filtering. Real-Time Imaging 1997;3:59-65.

[19] Van Vliet LJ, Young IT, Verbeek PW. Recursive Gaussian derivative filters. In: Proceedings of the 14th International Conference on Pattern Recognition, Brisbane, Australia, August 1998. p. 509-14.

[20] Young, I.T.; van Vliet, L.J.; van Ginkel, M., "Recursive Gabor filtering," Signal Processing, IEEE Transactions on [see also Acoustics, Speech, and Signal Processing, IEEE Transactions on], vol.50, no.11, pp. 2798-2805, Nov $2002 \mathrm{P}$

[21] Krzysztof Okarma Polynomial windows with low sidelobes' level Elsevier. Signal Processing Volume 87, Issue 4, April 2007, Pages 782-788

[22] Bracewell, R., The Fourier Transform and Its Applications. 3rd ed. New York: McGraw-Hill, 1999.

[23] Proc. Ieee Power Eng. Soc. Working Group 1433 Power Quality, [Online]. Available: http://grouper.ieee.org/groups/1433/

[24] Ribeiro. P. "Time-Varying Waveform Distortions in Power Systems" Wiley-IEEE Press (September 22, 2009) 